\title{
The hepatitis B vaccine originally jointly developed by China and the US is safe and effective
}

\author{
HOU YunDe \\ National Institute of Viral Disease Control and Prevention, Chinese Center for Disease Control and Prevention, Beijing 102206, China
}

Received February 16, 2014; accepted February 19, 2014; published online March 21, 2014 Citation: Hou YD. The hepatitis B vaccine originally jointly developed by China and the US is safe and effective. Sci China Life Sci, 2014, 57: 549-550, doi:
$10.1007 / \mathrm{s} 11427-014-4630-5$

According to the disease control and prevention data of China, hepatitis B vaccinations have caused 188 coincidence deaths of newborns totally from 2000 to 2013. No problems have been found with the vaccines. Besides inspection of the manufacturers, all vaccines are launched to the market after inspection of the central government of China. Similar events happened also in other vaccines. The recent deaths related to hepatitis $B$ vaccination have been proved to be coincidence. The hepatitis $\mathrm{B}$ virus vaccine used is originally jointly developed by the Chinese government and the US government. Its safety has been proved by the inoculation of one billion people around the globe.

Vaccines are major means for epidemic control. In 2009, during the new influenza A (H1N1) pandemic originating from Mexico, under the leadership of inter-departmental and inter-regional mechanisms for joint prevention and control, China implemented nationwide collaborative innovation, producing safe and effective vaccines within 87 days, which are unprecedented in the world, and inoculating 105 million people all over the country. Research showed that the protection rate of $\mathrm{H} 1 \mathrm{~N} 1$ vaccine reached $87.3 \%$, and China has established an inspection system for vaccine safety covering 70 million inoculated people, demonstrating the vaccines are safe and the Guillain-Barre syndrome has nothing to do with H1N1 vaccines. Moreover, it demonstrated for the first time that one dose of the vaccine is able to give effective protection. On 21 August 2009, Chen Zhu,

email: houyd20022003@aliyun.com the former minister of Ministry of Health of China, for the first time announced the aforementioned research results to the world in an international conference organized by Lancet in Beijing. Evaluation given by a third party, Tsinghua University, said that the timely production of the vaccine has been critical to the control of the pandemic, reducing 250 million infections and hospitalization of 69500 people.

In 1992, the hepatitis B surface antigen carries in China accounted for $9.75 \%$ of the Chinese population, making China a big country with a large population carrying hepatitis. On 1 January 1992, China implemented the hepatitis B immunization program; investigation in 2006 showed that the hepatitis B surface antigen carrier rate in the country has decreased to $7.18 \%$. This rate among children under five years old has been reduced to less than $0.5 \%$, meeting the requirement of the World Health Organization Western Pacific Region three years in advance.

In 2008, China included the prevention and control of viral hepatitis in a major science and technology project titled "Prevention and Treatment of AIDS and Viral Hepatitis and Other Major Infectious Diseases". During the "Eleventh Five-year Plan", researchers discovered that the non-response and low-response rates of $5 \mu \mathrm{g}$ hepatitis B vaccine were relatively high, and changed it to $10 \mu \mathrm{g}$, which was used in the widespread inoculation of 15-year-old teenagers in China in 2012. Afterwards, the high-dose vaccines of 20 and $60 \mu \mathrm{g}$ have been successfully developed. The plan for adult immunization has been carried out in the model area of the major science and technology project. It is 
believed that China will soon get rid of its title as a big country with a large population carrying hepatitis $\mathrm{B}$, and the
Chinese people will no longer have to suffer from this disease in the near future.

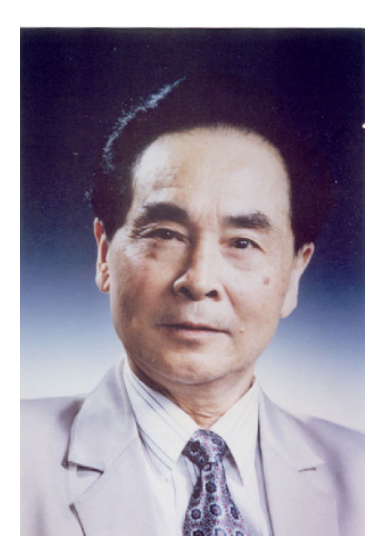

Biographical Sketch Hou YunDe, member and former vice president of the Chinese Academy of Engineering, was born in Changzhou, Jiangsu Province on July 13, 1929. He is a research fellow of the National Institute of Viral Disease Control and Prevention, Chinese Center for Disease Control and Prevention and director of the National Engineering Research Center for Viro Bio Technology, and holds the position of chief engineer of a major science and technology project titled "Prevention and Treatment of AIDS and Viral Hepatitis and Other Major Infectious Diseases". During the pandemic outbreak of the new H1N1 flu in 2009, as director of the Expert Committee of the Defense and Control Mechanism, he achieved success in the intervention of the flu, which is unprecedented in the human history and has been internationally recognized. Since 1986, he has been elected three times as chief scientist of the National High Technology Research and Development Program of China in the area of biotechnology. He has made outstanding achievements in molecular virology, genome project interferon, and control and prevention of major infectious diseases, and developed genome project interferon and other cytokines products, the first batch of high-tech products launched to the markets in China, among which interferon $\alpha 1 \mathrm{~b}$ is unprecedented around the globe. He has been elected twice as director of the International Society for Interferon Research. He has published more than 400 papers and seven books, won one first prize and seven second prizes of National Science and Technology Progress, 10 first prizes of Scientific and Technological Achievements of Ministry of Health, the Ho Leung Ho Lee Prize, and Medical Science Prize of China, and first won the title of Young and Middle-aged Scientists with Outstanding Contributions.

Open Access This article is distributed under the terms of the Creative Commons Attribution License which permits any use, distribution, and reproduction in any medium, provided the original author(s) and source are credited. 